"FOCUS ON" session:

Thrombosis and cardiovascular disease in women: epidemiology and risk factors 


\title{
Thrombophilia in young women candidate to the pill: reasons for and against screening
}

\author{
B. Cosmi, S. Coccheri \\ Division of Angiology, S.Orsola-Malpighi Hospital, University of Bologna, Italy
}

\section{Key Words}

Venous thromboembolism, thrombophilia, oral contraceptives, genetic testing

\begin{abstract}
Screening for thrombophilia in women candidate to the pill is still a matter of debate. Oral contraceptives may trigger venous thromboembolic events in carriers of common inherited thrombophilic defects. General screening is not cost-effective from an epidemiological point of view if the objective is to prevent death due to venous thromboembolism during oral contraception (OC). However, clinicians deal with single patients and personal and/ or family history for venous thromboembolism have limited value for identifying those women at risk of VTE complications during OC. A pharmacogenetics approach in prescribing $O C$ on the basis of each woman's genetic make-up could increase drug safety. A proper evaluation of the cost-effectiveness, the medical, psychosocial and legal consequences is needed before general screening with genetic testing for inherited thrombophilia can be recommended before OC.
\end{abstract}

Copyright $\odot 2002$ S. Karger AG. Basel
(๑) 2002 S. Karger AG. Basel

$1424-8832 / 02 / 0326-0315 \$ 18.50 / 0$

Fax +41613061234
The relative risk of venous thromboembolism (VTE) is increased by about 4-fold in users of oral contraceptives (OC), however the increase in the absolute risk is modest (from 1 case per 10.000 women years in non-pill users to 3 cases per 10.000 women years in pill users) [1]. It is estimated that more than 100 million women worldwide use OC. Although most OC users are healthy with a low background incidence of major disease, the large number of women who use OC worldwide means that even modest elevations in risk have the potential to affect a large number of women. OC can trigger VTE in carriers of unrecognised thrombophilic defects such as the R506Q mutation of factor $\mathrm{V}$ gene (FV Leiden) and the G20120A polymorphism in the prothrombin gene [2, 3, 4]. The estimated prevalence of these two defects ranges from 3$10 \%$ and $1-6 \%$ respectively in healthy Europeans. OC increase the risk of thrombotic complications in FV Leiden heterozygotes and homozygotes by 30-40-fold and 100-fold, respectively. OC increase the risk of cerebral vein thrombosis by 150 -fold during in the presence of the prothrombin mutation G20120A [5]. An interaction with a synergistic effect between OC and FV Leiden has been shown by Vandenbroucke et al.[1]. The increase in venous thromboembolism is about 4fold in OC users who are non carriers, about 7-fold in carriers of FV Leiden who do not use the pill and 35-fold in pill users who are also carriers of FV Leiden [1].

Pharmacogenetics is the study of the hereditary basis for differences in populations' response to drugs. Pharmacogenetics has the potential to allow physicians to choose drugs depending on the patient's genetic make-up with the aim

Benilde Cosmi MD

Division of Angiology, S.Orsola-Malpighi Hospital, University of Bologna,

Via Massarenti 9, 40138 Bologna Italy

Tel + 390516363420 - Fax: +39051341642

e-mail: bcosmi@med.unibo.it 
to increase efficacy and safety. Although screening for common inherited thrombophilic defects such as FV Leiden before OC could be useful to identify women at risk of VTE during OC, such screening is still a matter of debate. The problem can be approached by different perspectives: the epidemiologist's, the clinician's and the woman's. Epidemiologists have focused the discussion on the fatal complications of VTE such as pulmonary embolism and on the effect of FV Leiden and other rarer defects. It has been estimated that the screening of 200,000 women would be required to prevent one death by PE if the prevalence of defects such as FV Leiden was $10 \%$ in the general population [6].The case fatality rate of $1-2 \%$ among women who develop thrombosis during OC is considered negligible. In 1999 Palareti et al. [7] have shown that extensive thrombophilic screening before OC is not cost-effective. However, activated protein $\mathrm{C}$ resistance [APCR] assessment seems to have a favourable cost-effectiveness ratio. The alteration is frequent and has a synergistic effect with FV Leiden, sensibility and specificity of some methods are good. It has been shown that if all the costs associated with the treatment and long-term sequelae, such as the post-thrombotic syndrome, were considered, general screening would be effective if the cost of ACPR testing was less than 9 U.S. \$ [8].

However, the discussion has been limited to the interaction between FV Leiden and OC. The presence of the G20210A mutation of the prothrombin gene increases the risk of DVT by 16-50- fold and of cerebral vein thrombosis by 150 -fold in OC users vs. non users $[5,9,10]$. The prevalence of G20210A prothrombin polymorphism is estimated between $2-4 \%$ in the general population (more prevalent in Southern Europe). In 1999 Martinelli et al. [9] have shown an increased risk of VTE in carriers of G20120A prothrombin mutations. This mutation seems to have a synergistic effect with OC. The risk of VTE is 3 fold in the carriers who do not use OC, while it increases to 16-fold in carriers who use OC [9]. Legnani et al. have also shown that the G20120A prothrombin mutation has a synergistic interaction with OC [10]. The OR for VTE is almost 60 in carriers who also use $\mathrm{OC}$ versus non carriers and non users [10].

Cost-effectiveness of thrombophilia screening before OC has been calculated for FV Leiden and the rare thrombophilic defects. No data regarding cost-effectiveness of screening for G20120A prothrombin mutation are available. This type of screening would require genetic testing as no accurate functional assay is available for this mutation. Some authors therefore recommend selective screening in those women with personal and family history of VTE, in the attempt to increase cost-effectiveness and avoid denial of the benefits of OC to a large number of women $[11,12,13]$. However data from several studies indicate that family history of VTE has a limited value in the identification of carriers of common thrombophilic defects. Our group has evaluated the prevalence of thrombophilic defects according to family history of VTE in
479 thrombosis free women before OC [14]. A positive family history was present in $10 \%$ of the subjects. The prevalence of thrombophilic defects was similar in women with a negative and women with a positive family history for VTE. The sensitivity and positive predictive value of family history of VTE for thrombophilia was very low and unsatisfactory $(<50 \%)$. As a result, selective thrombophilia screening in women with a personal and family history of VTE can miss a relevant number of women at increased risk of thrombotic complications during OC. Indeed, a positive personal and/or family history of VTE could be considered by itself a contraindication to oral contraception regardless of any thrombophilic defect. Screening for thrombophilia could paradoxically be deemed irrelevant, but it still remains useful for specific assessment of the general thrombotic risk. The problem can arise in case of a negative personal and/or family history of VTE. Is it then necessary to search for thrombophilic defects? It may be not if we consider the problem from a merely epidemiological point of view, because universal screening is not cost-effective. The perspective may change if we considered not only the rare fatal cases, but also the burden of an episode of VTE and the related treatment, and of a possible post-thrombotic syndrome. There is considerable debate over the medical, psychosocial and legal consequences of screening for inherited thrombophilia and thus of genetic testing in asymptomatic individuals. It is usually assumed that such screening and especially genetic testing might have negative psychosocial, medical and legal consequences. The medical impact is negative in terms of being denied the access of effective and simple contraception, with the risk of unwanted pregnancies and their complications. However, the knowledge of an inherited thrombophilic predisposition has a positive impact for the possibility of preventing VTE in high risk situations and careful clinical surveillance. No proper evaluation of the medical impact of thrombophilia screening before OC in asymptomatic women has been conducted. The psychological impact may be negative with a decrease in asymptomatic subjects' quality of life and perception of well being. The social impact may be negative as well with asymptomatic individuals labelled as 'sick' because of an inherited defect with a higher risk of thrombosis. However, limited data on the psychosocial impact of inherited thrombophilia screening before $\mathrm{OC}$ in asymptomatic women are available and no data are available regarding women's preferences and perception of the problem of screening for inherited thrombophilia before OC.

Our group evaluated the emotional impact of thrombophilia screening in collaboration with the Department of Psychology of the University of Bologna [15]. 177 adults underwent thrombophilia screening with genetic testing (GT). They included male and females who were tested as patients after VTE, women before hormonal therapy and asymptomatic family members of thrombophilic subjects. A brief genetic counselling was given before blood sampling. They completed 
a validated questionnaire assessing: emotional state, perceived well being before and 20 days after receiving the results of GT for inherited thrombophilia. No significant harmful emotional effects of GT were detectable in the short term. An increase in perceived health status and well being and a decrease in sanitary fears and perceived life stress was observed in both the positive $(n=33)$ and negative $(n=104)$ group. Only the positive group decreased anxiety. The absence of adverse psychological reactions in this study might be associated to the specific testing programme that provided clear counselling about thrombophilia screening and the possibility of VTE prevention in high risk situations. However long term psychological effects were not evaluated.

The legal impact of genetic testing varies according to the health system organization and any 'screening' habits. Health insurance may be harder to obtain in certain countries. In other countries such as Italy, screening is recommended in all women before OC only as far as determination of ATIII. However, such screening is not cost-effective and does not evaluate the much more common defects.
The epidemiologists have correctly indicated that general screening is not cost-effective if the objective is to prevent death due to VTE during OC. The clinicians have to deal with single patients. No means other than personal and/ or family history and thrombophilia screening to identify those women at risk of VTE complications during OC are available so far. Moreover, nowadays the clinician often faces litigation issues which could arise from the identification of a thrombophilic defect only after an episode of VTE during OC in a woman to whom incomplete information had been given. Women are entitled to safe contraception. They cannot be denied an adequate and complete information a of the VTE risk associated with OC, and about the possibility of screening before OC. A pharmacogenetics approach in prescribing $\mathrm{OC}$ on the basis of each woman's genetic make-up could increase drug safety. A proper evaluation of the cost-effectiveness, the medical, psychosocial and legal consequences is needed before general screening for inherited thrombophilia can be recommended before OC.

\section{References}

1. Vandenbroucke JP, Koster T, Briet E, Reitsma $\mathrm{PH}$, Bertina RM, Rosendaal FR: Increased risk of venous thrombosis in oral contraceptive users who are carriers of factor V Leiden mutation. Lancet 1994; 344:1453-7.

2. Bertina RM, Koelemann BPC, Koster T, Rosendaal FR, Dirven RJ,de Ronde H, van der Vleden PA, Reitsma PH: Mutation in blood coagulation factor $\mathrm{V}$ associated with resistance to activated protein C. Nature 1994;369:64-7.

3. Poort SR, Rosendaal FR, Reitsma PH, Bertina RM: A common genetic variation in the 3'untranslated region of the prothrombin gene is associated with elevated plasma prothrombin levels and an increase in venous thrombosis. Blood 1996; 88:3698-3703.

4. Koster T, Rosendaal FR, de Ronde H, Briet E, Vandenbroucke JP, Bertina RM: Venous thrombosis due to poor anticoagulation response to activated protein C-Leiden Thrombophilia Study. Lancet 1993; 342:15036.

5. Martinelli I, Sacchi E, Landi G, Taioli E, Duca F, Mannucci PM: High risk of cerebral vein thrombosis in carriers of a prothrombin-gene mutation and in users of oral contraceptives. $\mathrm{N}$ Eng J Med 1998; 338:1793-7.

6. Vandenbroucke JP, van der Meer FJM, Helmerhorst FM, Rosendaal FR: Factor V Leiden: should we screen oral contraceptive users and pregnant women? BMJ 1996; 313:1127-30.

7. Palareti G, Legnani C, Frascaro M, Flamigni C, Gammi L, Gola G, Fuschini G, Coccheri S: Screening of activated protein $\mathrm{C}$ resistance before oral contraceptive treatment: a pilot study. Contraception 1999;59:293-9.

8. Vandenbroucke JP, Rosing J, Bloemenkamp KWM, Middeldorp S, Helmerhorst FM, Bouma BN, Rosendaal FR: Oral contraceptives and the risk of venous thrombosis. New Eng J Med 2001;344: 1527-35.

9. Martinelli I, Taioli E, Bucciarelli P, Akvahan S, Mannucci PM: Interaction between the G20120A mutation of the prothrombin gene and oral contraceptive use in deep vein thrombosis. Arterioscl Thromb Vasc Biol 1999; 19:700-3.

10. Legnani C, Palareti G, Guazzaloca G, Cosmi B, Lunghi B, Bernardi F, Coccheri S:Venous thromboembolism in young women: role of thrombophilic mutations and oral contraceptive use. Eur Heart J, 2002; 23:984-990.

11. Hannaford PC and Webb AMC on behalf of participants at an International Workshop: Evidence-Guided prescribing of combined oral contraceptives: consensus statement. Contraception 1996; 54:125-9.

12. McPherson K: Third generation oral contraception and venous thromboembolism. BMJ 1996; 312:68-9.

13. Walker ID: Factor V Leiden: should all women be screened prior to commencing the contraceptive pill? Blood Reviews 1999;13: 8-13.

14. Cosmi B, Legnani C, Bernardi F, Coccheri S Palareti G: Value of family history in indentifying women at risk of venous thromboembolism during oral contraception: observational study. BMJ 2001; 322: 1024-1025.

15. Razzaboni E, Gremigni P, Ricci Bitti PE, Legnani C, Palareti G: Emotional impact of genetic testing for inherited thrombophilia. B J Health Psychol, 2002, submitted 